\title{
Influence de la dose d'ammoniac et de la durée de traitement de fanes de pois chiche (Cicer arietinum $L$ ) sur les modifications biochimiques et l'estimation de la digestibilité de la matière organique
}

\author{
V Yaméogo, R Cordesse, M Inesta \\ ENSA-INRA, unité de zootechnie méditerranéenne, Place Pierre Viala, \\ 34060 Montpellier Cedex 01, France
}

(Reçu le 7 mars 1991; accepté le 28 mai 1991)

\begin{abstract}
Résumé - Une étude a été entreprise pour évaluer, dans des conditions de température $\left(54^{\circ} \mathrm{C}\right)$ et d'humidité $(20 \%)$ constantes, l'influence de doses d'ammoniac croissantes $(0,1,2,3$ et $5 \%$ de la matière sèche (MS)) sur la composition chimique : azote total (NT), azote soluble (NS), parois (NDF), composés phénoliques libérés par un traitement neutre (LAU 1) et un traitement alcalin (LAU 2) et la digestibilité de la matière organique des fanes de pois chiche estimée par la méthode des sachets avec des durés d'incubation dans le rumen de 6,24 et $48 \mathrm{~h}$ et par la méthode enzymatique décrite par Rexen (1977). Quatre durées de traitement étaient comparées (1, 3, 8 et $30 \mathrm{j}$ ). L'estimation de la digestibilité a été réalisée sur les échantillons qui ont présenté une bonne efficacité de traitement $\left(5 \%\right.$ de $\left.\mathrm{NH}_{3}\right)$. En parallèle, une paille de blé dur, variété "ardente" de caractéristiques connues, a été utilisée comme témoin. L'augmentation de la dose d'ammoniac entraîne une augmentation importante de la fixation de l'azote total et de l'azote soluble sur la paille de pois chiche. Cette fixation est restée supérieure à celle obtenue avec la paille de blé dur (16,6 contre $10,3 \mathrm{mg} / \mathrm{g}$ MS à la dose de $5 \%, P<0,01$ ). La durée de traitement n'a apporté d'amélioration notable que pour la fixation d'azote total et d'azote soluble et l'augmentation de cet accroissement est resté faible ( 0,5 à 1,2 points pour NT et 0,45 à 3,1 points pour NS). La dégradation des parois des fanes de pois chiche a été moins bonne que pour la paille de blé. La solubilité enzymatique et la dégradabilité in situ ont été accrues respectivement de 15,3 et 28,4 pour le pois chiche et de 19,4 et 41,0 points pour le blé.
\end{abstract}

fanes de légumineuses / $\mathrm{NH}_{3}$ / paramètre de traitement / dégradabilité in situ / solubilité enzymatlque / composition chimique

Summary - Changes in biochemical composition and in vitro organic matter digestibility assessed by of ammonla-treated chickpea fallen leaf (Cicer arletinum $\mathrm{L}$ ). Effects of ammonia rate and treatment duration. The effects of increasing ammonia rates $(0,1,2,3,5 \% D M)$ on biochemical changes and organic matter digestibility of ammonia-treated chickpea fallen leaf were studied. Four treatment durations were compared: 1, 3, 8, $30 \mathrm{~d}$. A well known durum wheat straw was used as control. A significant rise in the fixation rate of total nitrogen and soluble nitrogen was related to the increase in the amount of ammonia used. Total nitrogen fixation was higher for chickpea fallen leaf than for durum wheat straw ( +16.6 and $10.3 \mathrm{mg} / \mathrm{g} \mathrm{DM}$ respectively) the effect of the duration of treatment on nitrogen fixation is noticeable but weak. Enzyme solubility and in situ degradabi- 
lity were increased for chickpea straw ( $+18.3 \%$ and $19.4 \%)$ as for durum wheat straw $(+28.4 \%$ and + $41 \%)$. However, cell wall degradation was less significant in chickpea fallen leaf.

leguminous fallen leaf / ammonia treatment / in situ degradability / enzyme solubility / digestiblilty

\section{INTRODUCTION}

Les effets du traitement à l'ammoniac des pailles de céréales sont bien connus. De nombreux auteurs ont montré que ce traitement en modifiant les microstructures des pailles de céréales augmentait leur valeur énergétique et azotée et leur ingestibilité. Les fanes de légumineuses considérées comme ayant une meilleure valeur nutritive n'ont pas fait l'objet de traitements bien qu'elles constituent dans certains pays une ressource importante.

Le pois chiche (Cicer arietinum $L$ ), légumineuse cultivée pour sa graine, est surtout adapté aux zones sèches. II connaît ces dernières années un regain d'intérêt (Congrès de Saragosse, CIHEAM, CEE, ICARDA, 1988) qui permet d'espérer un essor de la culture et une disponibilité en grain et en fanes fortement accrue. Un traitement ammoniacal peut-il améliorer la valeur nutritive de ces fanes de légumineuses comme dans le cas des pailles de céréales ? L'objet de cette étude est de mesurer l'impact de ce traitement sur le niveau de fixation de l'azote et l'importance des dégradations des parois, facteurs d'augmentation de la digestibilité. Au travers des fanes de pois chiche c'est un modèle de légumineuse qui est également abordé.

\section{MATÉRIEL ET MÉTHODES}

L'étude a été réalisée avec des fanes de pois chiche, variété E36 du type gulabi, produit de sélection ENSA-INRA Montpellier, non encore inscrite et de la paille de blé dur de variété "Ardente" comme témoin.

Comme les conditions optimales de traitement des pailles de céréales sont bien connues (Solaiman et al, 1979; Waagepetersen et Vestergaard Thomsen, 1977; Cordesse, 1982 et 1989) le modèle expérimental a retenu comme variables: les paramètres dose d'ammoniac 10 , $1,2,3,5 \%$ par rapport à la matière sèche) et durée de traitement $(0,1,3,8$ et 30 j). La température $53-54^{\circ} \mathrm{C}$, l'humidité $20 \%$ ont été maintenues fixes. Pour la paille de blé dur témoin, seules les doses d' $\mathrm{NH}_{3} \mathrm{O}$ et $5 \%$ et les durées 0 , 8 et 30 j ont été testées.

Tableau I. Protocole expérimental.

Paramètres de traitement

Humidité

Température

Doses de $\mathrm{NH}_{3}$ en \% de la MS

Durée de traitement (j)

Nombre de répétitions

Nombres de dosages par répétition
Pois chiche

Blé dur

$20 \%$

$53-54{ }^{\circ} \mathrm{C}$

$0,1,2,3$ et 5

$53-54{ }^{\circ} \mathrm{C}$

$0,3,8$ et 30

0 et 5

2

2
0,8 et 30

2 
L'ammoniac a été apporté sous forme ionisée, en solution, à la concentration de $32 \%$ de $\mathrm{NH}_{3}$. L'eau de la solution ammoniacale a été prise en compte dans l'humidification des échantillons.

Les traitements sont réalisés avec des échantillons d'environ $100 \mathrm{~g}$ prélevés après homogénéisation sur un stock initial de $15 \mathrm{~kg}$ de fanes de pois chiche hachées. Ils sont humidifiés puis placés dans des flacons en verre hermétique (joint téflon), maintenus pendant le délai nécessaire dans une étuve réglée à 53$54^{\circ} \mathrm{C}$.

A l'issue des différents traitements, les échantillons sont aérés pendant $5 \mathrm{j}$, broyés (grille de $1 \mathrm{~mm}$ ), stockés en boîtes étanches avant analyse.

\section{Mesures chimiques et biologiques}

Les fractions azotées totales et solubles sont dosées selon la technique colorimétrique de Nessler (Umbreit et al, 1959) après minéralisation (méthode Kjeldahl) pour l'azote total et après extraction selon la méthode décrite par Vérité et Demarquilly (1978) pour l'azote soluble.

Les parois végétales sont dosées par la méthode Van Soest et Wine (1967).

Le degré de lignification estimé selon la technique Lau et van Soest (1981) permet de mesurer successivement les composés phénoliques libérés dans un tampon neutre (LAU 1), ceux libérés après traitement alcalin fort (LAU 2). Les résultats exprimés en densité optique (DO) à $280 \mathrm{~nm}$ sont proportionnels à la concentration en acides phénoliques extraits.

La solubilité enzymatique est estimée selon la méthode de Rexen (1977). La dégradabilité in situ a été réalisée sur des échantillons non traités et traités à la dose de $5 \%$ pendant une durée de $8 \mathrm{j}$. Quatre moutons porteurs d'une fistule du rumen et recevant un régime standard contenant $13 \%$ de matières azotées totales (MAT) ont été utilisés (Michalet-Doreau et al, 1987).

Les résultats ont été soumis à une analyse de variance simple (Snedecor et Cochran, 1968).

\section{RÉSULTATS}

En l'état, les fanes de pois chiche (tableau Ii) sont plus riches en azote que les pailles de blé dur $(0,54$ contre $0,47 \%)$ et moins riches en parois ( 78 contre $85 \%$ ). La teneur en lignine est toutefois plus élevée chez le pois chiche $(15,5 \pm 0,6$ vs $5,9 \pm$ $0,2)$.

Après une durée de 8 j (tableau II) les effets du traitement varient en fonction des doses utilisées.

- La quantité d'azote fixée s'accroît fortement entre les traitements 0 et $3 \%$ d' $\mathrm{NH}_{3}$ $(2,1 \%$ vs $0,54 \%)$, plus modérément ensuite jusqu'à $5 \%$ testé $(2,2$ vs $2,1 \%)$.

Les valeurs de NDF, LAU 1, LAU 2, varient peu pour des doses de 1,2 et $3 \%$ $\mathrm{d}^{\prime} \mathrm{NH}_{3}$, davantage avec un traitement à $5 \%$.

- La comparaison des effets du traitement à la dose de $5 \%$ sur les fanes de pois chiche et les pailles de blé dur met en évidence une fixation plus importante d'azote pour les fanes de pois chiche (respectivement $+1,66$ et $+1,03$ points pour l'azote total) mais une dégradation plus importante des parois de la paille de blé dur. La teneur en NDF diminue de 8 points au lieu de 5 et celle des acides phénoliques libérés avec le tampon neutre de 750 au lieu de 250.

À la température utilisée, les réactions de fixation de l'azote, de libération des composés phénoliques et de dégradation des composés pariétaux sont rapides pour les fanes de pois chiche et atteignent leur valeur maximale après $3-8 \mathrm{j}$ de traitement. Pour les échantillons de paille de blé dur, les modifications biochimiques sont maximales après 8 j de traitement.

La solubilité enzymatique des produits non traités, (tableau III) est plus importante pour les fanes de pois chiche $(39 \%)$ que 
Tableau II. Modifications biochimiques de la paille de blé dur et de la paille de pois chiche après 8 jours de traitement avec différentes doses d'ammoniac et signification statistique des différences (3) (Résultats exprimés en \% de la MS).

\begin{tabular}{|c|c|c|c|c|c|c|c|c|c|}
\hline \multirow[b]{2}{*}{ Doses de $\mathrm{NH}_{3}$} & \multicolumn{6}{|c|}{ Pailles de pois chiche } & \multicolumn{3}{|c|}{ Pailles de blé dur } \\
\hline & 0 & 1 & 2 & 3 & 5 & $5-0(1)$ & 0 & 5 & $5-0(1)$ \\
\hline MM & 5,1 & $5,3^{*}$ & 5,3 & 5,2 & 5,1 & - & 7,7 & 7,1 & - \\
\hline MO & 94,9 & $94,7^{\star}$ & 94,7 & 94,8 & 94,9 & - & 92,4 & 92,9 & - \\
\hline NT & 0,54 & $11,7^{* *}$ & $1,8^{* *}$ & $2,1^{\star \star}$ & 2,2 & $+1,66$ & 0,47 & $1,50^{* *}$ & $* \quad+1,03$ \\
\hline NS & 0,30 & $0,70^{* *}$ & * $1,18^{* *}$ & * $1,31 * *$ & $1,55^{\star *}$ & $+1,25$ & 0,16 & $0,86^{* *}$ & $+0,7$ \\
\hline NDF & 78 & 77 & 76 & $75^{\star}$ & $73^{*}$ & -5 & 85 & $77^{\star \star \star}$ & -8 \\
\hline Cellulose & 45 & - & - & - & 45,5 & $+0,5$ & 42,7 & 44,1 & $+1,4$ \\
\hline Hémicellulose & 18,2 & - & - & - & 14,2 & -4 & 30,8 & 22,6 & $-8,2$ \\
\hline Lignine & 15,8 & - & - & - & 15,7 & - & 11,0 & 10,7 & - \\
\hline LAU 1(2) & 459 & 608 & 589 & $615^{\star *}$ & $749^{* *}$ & +250 & 584 & $1336^{* *}$ & +752 \\
\hline LAU 2(2) & 352 & 350 & 352 & 339 & 300 & -52 & 1743 & $1263^{*}$ & -480 \\
\hline
\end{tabular}

(1) Variation dose 5 moins dose 0; (2) Pour les LAU 1 et LAU 2 les résultats sont exprimés en DO/g MS; (3) Signification statistique des différences enregistrées entre chacun des traitements et le témoin au seuil de $5 \%\left(^{*}\right)$, au seuil de $1 \%\left(^{* *}\right)$.

pour la paille de blé $(28,5 \%)$. La solubilité enzymatique des échantillons traités pendant $8 \mathrm{j}$ à la dose de $5 \%$, est en revanche très voisine (54 et $57 \%$ ).

La dégradabilité in situ (tableau IIlb) conduit à des résultats assez comparables. Après un séjour de $6 \mathrm{~h}$ dans le rumen, la dégradabilité est supérieure pour les fanes de pois chiche non traitées, mais devient identique pour les 2 espèces après un séjour de $48 \mathrm{~h}$. Par contre, sur les échantillons traités, malgré une dégradabilité à $6 \mathrm{~h}$ plus importante pour les fanes de pois chiche $(25,3$ vs 18,0$)$, celle enregistrée à $48 \mathrm{~h}$ est en faveur de la paille de blé ( 59 vs $45 \%$ ). L'efficacité du traitement calculé après $48 \mathrm{~h}$ de séjour des échantillons traités et non traités montre un gain de 7,3 points pour les fanes de pois chiche et de 22,2 points pour la paille de blé dur. Entre la $6^{e}$ et la $48^{\circ} \mathrm{h}$ de séjour dans le rumen, la dégrada- tion de la matière organique dépend essentiellement de l'action de la microflore. II est possible de constater (tableau IIIb), en comparant les 2 durées de séjour, que le traitement alcalin n'améliore pas la dégradabilité des fanes de pois chiche $(21,0$ vs $19,4)$ alors qu'il l'augmente de manière importante pour la paille de blé dur $(27,4$ vs $41,0)$.

\section{DISCUSSION ET CONCLUSION}

Le traitement à l'ammoniac a un effet significativement $(P<0,01)$ positif sur les teneurs en azote total et azote soluble, quelle que soit la dose d' $\mathrm{NH}_{3}$ (tableau II). Ce résultat est conforme à ceux relevés dans la bibliographie pour de nombreuses pailles (Sundstol et al, 1978; Kiangi et al, 1981; Borhami et Sundstol, 1982; Guillermin et al, 1988). 
Tableau III. Solubilité enzymatique et dégradabilité des fanes de pois chiche et des pailles de blé dur traitées ou non avec l'ammoniac.

$\begin{array}{ccc}\begin{array}{c}\text { Fanes ou } \\ \text { pailles }\end{array} & \begin{array}{c}\text { Fanes ou } \\ \text { pailles }\end{array} & \begin{array}{c}\text { Variation } \\ 2-1\end{array} \\ \text { non traitées } & \text { traitées } \\ \text { (1) } & \text { (2) }\end{array}$

a- Solubilité enzymatique en \% MO

\begin{tabular}{lrrr} 
PC & 38,7 & 54,0 & $+15,3$ \\
BD & 28,5 & 56,9 & $+28,4$ \\
\multicolumn{4}{c}{ b- Dégradabilité } \\
\multicolumn{4}{c}{ in situ en \% MS } \\
PC 6 h & 16,4 & 25,3 & $+8,9$ \\
24 h & 28,8 & 38,5 & $+9,7$ \\
48 h & 37,4 & 44,7 & $+7,3$ \\
Variation 48-6 h & & & \\
BD 6 h & 9,3 & 18,0 & $+8,7$ \\
24 h & 25,5 & 42,8 & $+17,3$ \\
48 h & 36,8 & 59,0 & $+22,2$ \\
& & & \\
Variation 48-6 h & $+27,5$ & $+41,0$ & \\
\hline
\end{tabular}

PC: fanes de pois chiche; BD : pailles de blé dur.

Le pourcentage d'azote fixé par rapport à l'azote injecté au cours du traitement a été plus élevé pour la paille de pois chiche, 67 et $40,3 \%$ respectivement pour les doses $3 \%$ et $5 \%$ pour la paille de blé dur, $25 \%$ seulement, valeurs supérieures à celles observées par Kiangi et al (1981), Dulphy et al (1984); Grenet et Demarquilly (1987); Chenost (1989). Sundstol et al (1978) indiquent, sans donner la dose d'ammoniaque appliquée, les valeurs de 0,91 et $2,47 \mathrm{~g}$ d'azote par $\mathrm{kg}$ de paille de haricot avant et après traitement, soit une efficacité de fixation de $63 \%$ si la dose d'ammoniac était de $3 \%$ ou $38 \%$ pour une dose de $5 \%$.
Les paramètres biochimiques NDF et LAU1 ne sont pas modifiés aux faibles doses d'ammoniac ( 1 et $2 \%$, cf tableau II). Les doses plus élevées entraînent en revanche des modifications significatives, moins importantes toutefois que celles enregistrées avec la paille de blé. Des concentrations en ions $\mathrm{NH}_{4}{ }^{+}$assez élevées sont donc nécessaires pour déclencher la dégradation des parois végétales. Ce résultat est confirmé dans de nombreux rapports (Waagepetersen et Vestergaard Thomsen, 1977; Hartley et Jones, 1978; Sundstol et al, 1979) et, plus récemment, par Schneider et Flachowsky (1990) dans une étude plus complète où sont prises en compte 2 températures, $3 \mathrm{du}$ rées, 3 concentrations en humidité, 3 doses d'NH $\mathrm{NH}_{3}$.

La comparaison des résultats de solubilité enzymatique (tableau IIla) et de dégradabilité in situ (tableau IIIb) montre que les fanes de pois chiche sont au départ plus digestibles que celles de blé dur. Le traitement ammoniacal n'augmente la solubilité enzymatique des fanes de pois chiche que de 15 points contre 28 points pour la paille de blé dur. Les résultats en sachets de nylon conduisent à la même observation. De même, la solubilité des composés phénoliques à l'extraction alcaline (LAU 2) va dans le même sens. Ces résultats sont en accord avec ceux de Morrison (1974), van Soest (1981), Mason et al (1988).

À notre connaissance, les références bibliographiques sur la digestibilité des fanes de légumineuses traitées à l'ammoniac et, plus particulièrement, sur celles de pois chiche, n'existent pas. Par contre, les estimations de digestibilité sur les pailles de céréales (solubilité enzymatique, dégradabilité, digestibilité in vivo ou in vitro) sont nombreuses et toutes corroborent nos résultats. Les études de Jarrige et Thivend (1969) ont montré qu'à digestibilité de MS égalẻ entre légumineuses et graminées, la 
cellulose Weende des légumineuses était plus résistante que celle des graminées. La différence de modifications biochimiques des pailles testées dans cette étude pourrait s'expliquer par la plus grande résistance de la lignine des légumineuses aux hydrolyses alcalines montrée par Gordon (1975) et van Soest (1981). II en résulte que, malgré une fixation d'ammoniac importante, les structures pariétales des tanes de pois chiche sont peu modifiées par le traitement alcalin; l'augmentation de la valeur nutritive est donc faible.

D'un point de vue économique, le traitement alcalin est moins justifié avec les fanes de pois chiche qu'avec les pailles de céréales. Des études complémentaires avec d'autres fanes de légumineuses devraient être entreprises.

\section{RÉFÉRENCES}

Borhami BEA, Sundstol F (1982) Studies on ammonia-treated straw. I. The effects of type and level of ammonia, moisture content and treatment time on the digestibility in vitro and enzyme soluble organic matter of oat straw. Anim Feed Sci Technol 7, 45-51

Chenost M (1989) Intérêt comparé du traitement à l'ammoniac et d'une complémentation appropriée de pailles de blé (niveau et nature des compléments énergétiques et azotés) pour l'alimentation de génisses de race laitière de deux ans en croissance hivernale modérée. Ann Zootech 38, 29-47

Cordesse R (1982) Amélioration de la valeur nutritive des pailles par les traitements chimiques. Thèse Dr Ingénieur, Montpellier, $99 p$

Cordesse R (1989) Valorisation alimentaire des résidus de récolte par le traitement à l'ammoniac. Aspect biochimique et technologique. In: Pâturages et alimentation des ruminants en zone tropicale humide (Xande $\mathrm{A}$, Alexandre G, eds) 427-440, INRA, Paris

Dulphy JP, Boissau JM, Leblanc F (1984) Influence du traitement à l'ammoniac sur la valeur alimentaire de pailles distribuées à des génisses. Bul Tech CRVZ Theix, INRA, 55, 25-32

Gordon AJ (1975) A comparison of some chemical and physical properties of alkali lignins from grass and lucerne hays before and after digestion by sheep. J Sci Food Agric 26, 1551-1559

Grenet E, Demarquilly C (1987) Rappels sur la digestion des fourrages dans le rumen (parois) et ses conséquences. In: Les fourrages secs : récolte, traitement, utilisation (Demarquilly $C$, ed) INRA, Paris, 141-162

Guillermin P, Cordesse R, Dulphy JP (1988) Prévision de la digestion des pailles et des foins de graminées traités ou non à l'ammoniac à partir de mesures de laboratoire. Ann Zootech 34, 43-56

Hartley RD, Jones EC (1978) Effect of aqueous ammonia and other alkalis on the in vitro digestibility of barley straw. $J$ Sci Food Agric 29, 92-98

Jarrige $R$, Thivend $P$ (1969) Action d'une cellulase fongique sur les membranes et son intérêt pour prévoir la digestibilité des plantes fourragères. Ann Biol Anim Biochim Biophys $9,171-190$

Kiangi EMI, Kategile JA, Sundstol F (1981) Different sources of ammonia for improving the nutritive value of low quality roughages. Anim Feed Sci Technol 6, 377-386

Lau MM, van Soest PJ (1981) Titrable groups and soluble phenolic compounds as indicators of the digestibility of chemically treated roughages. Anim Feed Sci Technol 6, 123131

Mason VC, Hartley RD, Keene AS, Corby JM (1988) The effect of ammoniation on the nutritive value of wheat barley and oat straws. 1. Changes in chemical composition in relation to digestibility in vitro and cell wall degradability. Anim Feed Sci Technol 19, 159-171

Michalet-Doreau $B$, Vérité $R$, Chapoutot $P$ (1987) Méthodologie de mesure de la dégradabilité in sacco de l'azote des aliments dans le rumen. Bull Tech CRVZ Theix, INRA, 69, 5-7

Morrison IM (1974) Structural investigations on the lignin carbohydrate complex of lolium perenne. Biochem J 139, 197-204

Rexen B (1977) Enzyme solubility. A method for evaluating the digestibility of alkali-treated straw. Anim Feed Sci Technol 2, 205-218 
Schneider M, Flachowsky G (1990) Studies on ammonia treatment of wheat straw: effects of level of ammonia, moisture content, treatment time and temperature on straw composition and degradation in the rumen of sheep. Anim Feed Sci Technol 29, 251-264

Snedecor GW, Cochran WG (1968) Expérience factorielle. In: Méthodes statistiques. Association de Coordination des Techniques Agricoles, MNE, Rue de Bercy, Paris, 379-451

Solaiman SG, Horn GW, Owens FN (1979) Ammonium hydroxyde treatment on wheat straw. J Anim Sci 49, 802-808

Sundstol F, Coxworth E, Mowat DN (1978) Amélioration de la valeur nutritive de la paille par le traitement à l'ammoniac. Rev Mond Zootech 26, 13-21

Sundstol F, Said AN, Arnason J (1979) Factors influencing the effect of chemical treatment on the nutritive value of straw. Acta Agric Scand 29, 179-190

Umbreit WW, Burris RH, Stauffer JF (1959) Manometric methods. Burgess Publ 238-274

Van Soest PJ (1981) Limiting factors in plant residues of low biodegradability. Agric Environ $6,135-143$

Van Soest PJ, Wine RH (1967) Use of detergents in the analysis of fibrous feeds. IV. Determination of plant cell-wall constituents. $J$ Assoc Off Agric Chem 50, 50-55

Vérité R, Demarquilly C (1978) Qualité des matières azotées des aliments pour ruminants. In: “La vache laitière», Ed INRA, Paris, 143157

Waagepetersen J, Vestergaard-Thomsen $\mathrm{K}$ (1977) Effect of digestibility and nitrogen of barley straw of different ammonia treatments. Anim Feed Sci Technol 2, 131-142 